\title{
ENDOVASCULAR STENT-GRAFT REPAIR OF THORACIC AORTIC ANEURYSMS
}

R. Scott Mitchell, $\mathrm{MD}^{\mathrm{c}}$

Michael D. Dake, MD ${ }^{\mathrm{a}}$

Charles P. Semba, MD ${ }^{a}$

Thomas J. Fogarty, MD ${ }^{\mathrm{b}}$

Christopher K. Zarins, $\mathrm{MD}^{\mathrm{b}}$

Robert P. Liddell, BA ${ }^{a}$

D. Craig Miller, $\mathrm{MD}^{\mathrm{c}}$
Conventional repair of aneurysms of the descending thoracic aorta entails thoracotomy and graft interposition. For elderly patients and those with previous operations, obesity, respiratory insufficiency, or other comorbidities, such a procedure entails significant mortality and morbidity. Transluminal stent-graft placement offers an alternative approach with potentially less morbidity and quicker recovery; however, the effectiveness and durability of stent-grafts remain uncertain. Methods: Since July 1992, thoracic aortic stent-grafts have been placed in 44 patients with a variety of pathologic conditions. Each graft was individually constructed from selfexpanding, stainless-steel $Z$ stents covered with a woven Dacron polyester fabric graft. Graft dimensions were determined from spiral computed tomographic scans. All implants were performed in the operating theater under fluoroscopic and transesophageal echocardiographic guidance. Follow-up was by computed tomography and contrast angiography in all cases. Patient data: There were 36 men and 8 women. Mean age was 66 years (range 35 to 88 years). Mean aneurysmal diameter was $6.3 \mathrm{~cm}$ (range 4.0 to $9.4 \mathrm{~cm}$ ). Etiologies included 23 degenerative aneurysms, four posttraumatic aneurysms, four pseudoaneurysms, and one chronic aortic dissection. Results: There were three early deaths ( $<30$ days) and two late deaths. One early death resulted from graft failure. There were two instances of paraparesis or paraplegia, with one associated early death. A single stent was deployed in 27 patients, two stents were required in 14 patients, and three stents were required in three patients. In 23 patients, vascular access was attained through the femoral artery; abdominal aortic access, either native or graft, was necessary in the remaining 21 patients. Twelve grafts were placed in conjunction with open abdominal aortic surgical procedures. Mean follow-up (98\% complete) was 12.6 months (range 1 to 34 months). One late death occurred from aneurysmal expansion and rupture in a patient with a persistent midgraft leak. The second late death may have resulted from aneurysmal rupture. Immediate thrombosis was achieved in 36 patients, and late thrombosis was achieved in three others. Failure to achieve complete aneurysmal thrombosis occurred in five patients, however, and one individual (previously noted) died of aneurysmal expansion and rupture; the remaining four are being carefully monitored. Only one patient has required conversion of the stent to an open procedure; a contained rupture of the false lumen of a chronic dissection eventually necessitated total descending thoracic aortic exclusion. Conclusions: These early results support the hypothesis that endovascular stent-graft place-
From the Department of Radiology, ${ }^{a}$ the Division of Vascular Surgery, ${ }^{b}$ and the Department of Cardiovascular Surgery, ${ }^{c}$ Stanford University School of Medicine, Stanford, Calif.

Read at the Twenty-first Annual Meeting of The Western Thoracic Surgical Association, Coeur d'Alene, Idaho, June 21-24, 1995.

Received for publication June 21, 1995; revisions requested Sept. 21, 1995; revisions received Oct. 24, 1995; accepted for publication Nov. 8, 1995.
Address for reprints: R. Scott Mitchell, MD, Department of Cardiovascular Surgery, Stanford University School of Medicine, Falk Cardiovascular Research Center, Stanford, CA 94305-5247.

J Thorac Cardiovasc Surg 1996;111:1054-62

Copyright (c) 1996 by Mosby-Year Book, Inc.

$0022-5223 / 96 \$ 5.00+0 \quad \mathbf{1 2 / 6 / 7 0 5 4 0}$ 
ment may be a safe and durable treatment for selected patients with aneurysmal disease of the descending thoracic aorta. Large introducer size (26F outer diameter) and relatively limited angulation capability, as well as imprecise deployment techniques, currently limit its applicability. Distal embolization and stent migration have not been observed. Failure to achieve complete aneurysmal thrombosis may allow continued aneurysmal expansion and rupture. Further follow-up is clearly necessary to evaluate the true long-term effectiveness of this procedure. (J Thorac Cardiovasc Surg 1996;111:1054-62)

$\mathrm{P}$ atients with aneurysms of the thoracic aorta continue to present important clinical problems. There appears to be an increase in the absolute number of aneurysms, which may reflect the increased life expectancy of the population or perhaps may reflect improved diagnostic capabilities. Since the first reports of homograft repair of thoracic aortic aneurysms by Swan and coworkers ${ }^{1}$ in 1950 and DeBakey and Cooley ${ }^{2}$ in 1953, aneurysmal repair has become a widely accepted treatment modality. Nevertheless, these procedures are still associated with significant morbidity, chiefly related to major thoracotomy, use of cardiopulmonary bypass with its attendant anticoagulation in many cases, and postoperative complications including bleeding, paraplegia, stroke, renal insufficiency, and the necessity for prolonged ventilatory support. Since July 1992, we have experimented with an endovascular stent-graft repair technique for descending thoracic aortic aneurysms in an effort to minimize such morbidities.

The incidence of thoracic aortic aneurysms is estimated to be approximately 6 cases $/ 100,000$ person-years. ${ }^{3}$ The afflicted population is usually elderly and predominantly male. Frequent comorbidities include hypertension, coronary artery disease, obstructive pulmonary disease, and congestive heart failure, all of which have significant impact on recovery from operative repair. Concomitant abdominal aortic aneurysms have been estimated to occur in $10 \%$ to $20 \%$ of these patients. Although the natural history of patients with thoracic aortic aneurysms is not so well defined as that of patients with abdominal aortic aneurysms, Pressler and $\mathrm{McNamara}^{4}$ reported the outcome in 22 patients treated medically: $40 \%$ died of progressive aneurysmal dilatation and rupture and $32 \%$ died of other cardiovascular disease. The actuarial 1- and 5-year survivals for patients not operated on have been estimated to be $60 \%$ and $20 \%$, respectively. ${ }^{3,4}$

Most aneurysms of the thoracic aorta are ath- erosclerotic or degenerative in etiology. Other causes include mycotic aneurysms, posttraumatic false aneurysms, and aneurysms of the ductus diverticulum. Recent experience has allowed some understanding of the evolution from aortic intramural hematomas and giant penetrating ulcers to the formation of thoracic aortic aneurysms. The role of hypertension is paramount, contributing to the increased wall tension as well as causing degenerative changes in the medial cell layers. Increased size also causes an increase in wall tension, with further dilatation and eventual rupture.

The traditional therapy for patients with thoracic aortic aneurysms is surgical repair. Although no precise predictive criteria exist, most surgeons recommend repair of any symptomatic aneurysm or any aneurysm whose dimension exceeds one and one half to two times the transverse dimension of an adjacent normal aortic segment, providing the patient is a reasonable candidate for the operation. Although there have been significant advances in the intraoperative and postoperative management of these elderly and frequently debilitated patients, operative mortality ranges from 5\% to $20 \%$ in large series, ${ }^{5-7}$ and postoperative renal and pulmonary insufficiency frequently results in prolonged hospital stays.

Transluminally placed endovascular stent-grafts are an attractive alternative that may potentially reduce operative risk and perioperative morbidity. The success of the first endovascular repairs of abdominal aortic aneurysms ${ }^{8}$ prompted our investigation into the feasibility of thoracic aortic aneurysmal repairs with transluminally placed endovascular stent-grafts. This report expands on our initial experience with 13 patients ${ }^{9}$ to our current expericnce of 44 patients operated on during a 34-month period, documents the mean follow-up of 12.6 months (range 1 to 34 months), and, perhaps most importantly, allows some recommendations from our admittedly steep learning curve. 


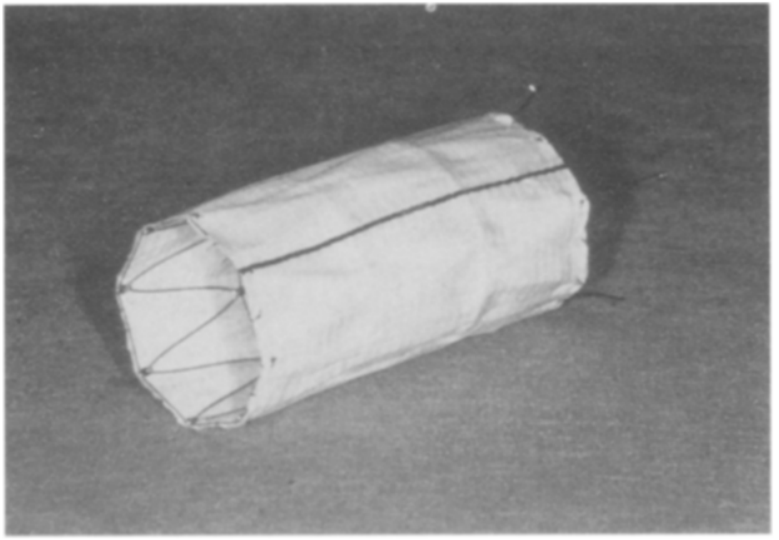

Fig. 1. Dacron polyester fabric graft covers a series of self-expanding $Z$ stents.

\section{Methods}

Between July 1992 and May 1995, under an investigational protocol approved by the Institutional Review Board of the Stanford University Medical Center, 44 patients underwent transluminal endovascular stent-graft repair of thoracic aortic aneurysms. Informed consent was obtained from every patient before operation, and all patients were monitored after operation according to an approved imaging protocol (Table I). All procedures were performed in the operating theater with the patient under general anesthesia, with monitoring by a portable digital radiographic $\mathrm{C}$-arm image intensifier, transesophageal echocardiography, and invasive radial and pulmonary artery monitoring. Intraoperative angiography was performed in all cases, initially to localize the aneurysm and subsequently to confirm exclusion of the aneurysm after stent-graft deployment. Patients were positioned and draped sterilely for a lateral thoracotomy, a double-lumen endotracheal tube was inserted in most cases, and cardiopulmonary bypass standby was available for every case.

Endovascular prosthesis. The endovascular stent-graft was individually constructed for each patient and was gas sterilized before use. In general, the stent-graft was oversized by 2 to $3 \mathrm{~mm}$ to allow a continued radial expansile force and an effective friction seal. A stainless-steel endoskeleton was constructed with a series of self-expanding Z-shaped stents "box-carred" together. A standard Cooley VERI-SOFT woven Dacron polyester fabric graft (Meadox Medicals, Inc., Oakland, N.J.) with its crimps ironed out was attached to the stent by a series of 5-0 interrupted polypropylene sutures (Fig. 1). The diameter and length of the stent-graft were determined for each patient from measurements obtained from a preoperative spiral computed tomographic scan. The device was then radially compressed and loaded into a $26 \mathrm{~F}$ (outer diameter) Teflon (polytetraffuoroethylene) sheath.

Endovascular procedure. With the patient under general anesthesia, the femoral artery, iliac artery, or abdominal aorta was surgically exposed. After precise localization of the aneurysmal neck by means of intraoperative
Table I. Imaging protocol

\begin{tabular}{lccc} 
& $\begin{array}{c}\text { Chest } \\
\text { radiography* }\end{array}$ & $\begin{array}{c}\text { Spiral } \\
C T\end{array}$ & Arteriography \\
\hline Before procedure & $\mathrm{x}$ & $\mathrm{x}$ & $\mathrm{x}$ \\
During procedure & & $\mathrm{x}$ & $\mathrm{x}$ \\
After procedure & $\mathrm{x}$ & $\mathrm{x}$ & $\mathrm{x}$ \\
2 mo after procedure & $\mathrm{x}$ & & \\
6 mo after procedure & $\mathrm{x}$ & $\mathrm{x}$ & $\mathrm{x}$ \\
1 yr after procedure & $\mathrm{x}$ & $\mathrm{x}$ & $\mathrm{x}$ \\
2 yr after procedure & $\mathrm{x}$ & $\mathrm{x}$ & \\
$\quad$ & & & \\
\hline and annually thereafter & & & \\
CT, Computed tomography. & & & \\
* Posteroanterior and lateral chest radiographs. &
\end{tabular}

angiography and transesophageal echocardiography, the $26 \mathrm{~F}$ (outer diameter) Teflon sheath with an indwelling obturator-dilator was introduced into the aortic arch over a superstiff 0.038 inch guide wire. After it traversed the aneurysm the obturator was removed, leaving the sheath proximal to the proximal aneurysmal neck, and the stentgraft was loaded from its cartridge and advanced into the sheath under fluoroscopic guidance.

Just before deployment, mean systemic arterial pressure was lowered to approximately $50 \mathrm{~mm} \mathrm{Hg}$ with intravenous vasodilators and $\beta$-blockers in an attempt to minimize distal migration of the graft during deployment. After final positioning, stent-graft deployment was accomplished by rapidly withdrawing the sheath from around the self-expanding stent-graft. Completion angiography was then performed to confirm proper positioning, and additional stent-grafts were placed proximally or distally as necessary to ensure aneurysmal exclusion. The sheath was then removed, the artery was repaired, and the patient was returned to the intensive care unit. Occasionally, a large $(25 \mathrm{~mm})$ angioplasty balloon was used to improve stent position or expansion. Total elimination of contrast enhancement of the aneurysmal sac (Fig. 2) was the desired objective in all cases.

Patient population. These 44 patients had a heterogenous mix of pathologies and risk factors. During the same interval, 60 patients had conventional open repair of descending thoracic aortic aneurysms or dissections. In our early experience, the first four patients were all deemed surgical candidates. Soon thereafter, patients considered not to be operative candidates for a variety of pulmonary, cardiac, or other problems (Table II) were enrolled in this clinical experiment. There were 36 men and eight women. Mean age was 66 years (range 35 to 88 years), and mean aneurysmal diameter was $6.3 \mathrm{~cm}$ (range 4.0 to $9.4 \mathrm{~cm}$ ). Etiologies included atherosclerosis in 24 patients, intramural hematoma or penetrating ulcer in eight, chronic posttraumatic origin in four, pseudoaneurysm in four, ductus diverticular aneurysm in four, and chronic aortic dissection in one. The mean stent-graft diameter was $3.5 \mathrm{~cm}$ (range 2.4 to $4.5 \mathrm{~cm}$ ), and the mean length of the device was $10.2 \mathrm{~cm}$ (range 5.0 to $18.0 \mathrm{~cm}$ ).

The femoral artery was the most common site for insertion; it was used in 21 patients. Twelve patients underwent concomitant abdominal aortic aneurysm repair 

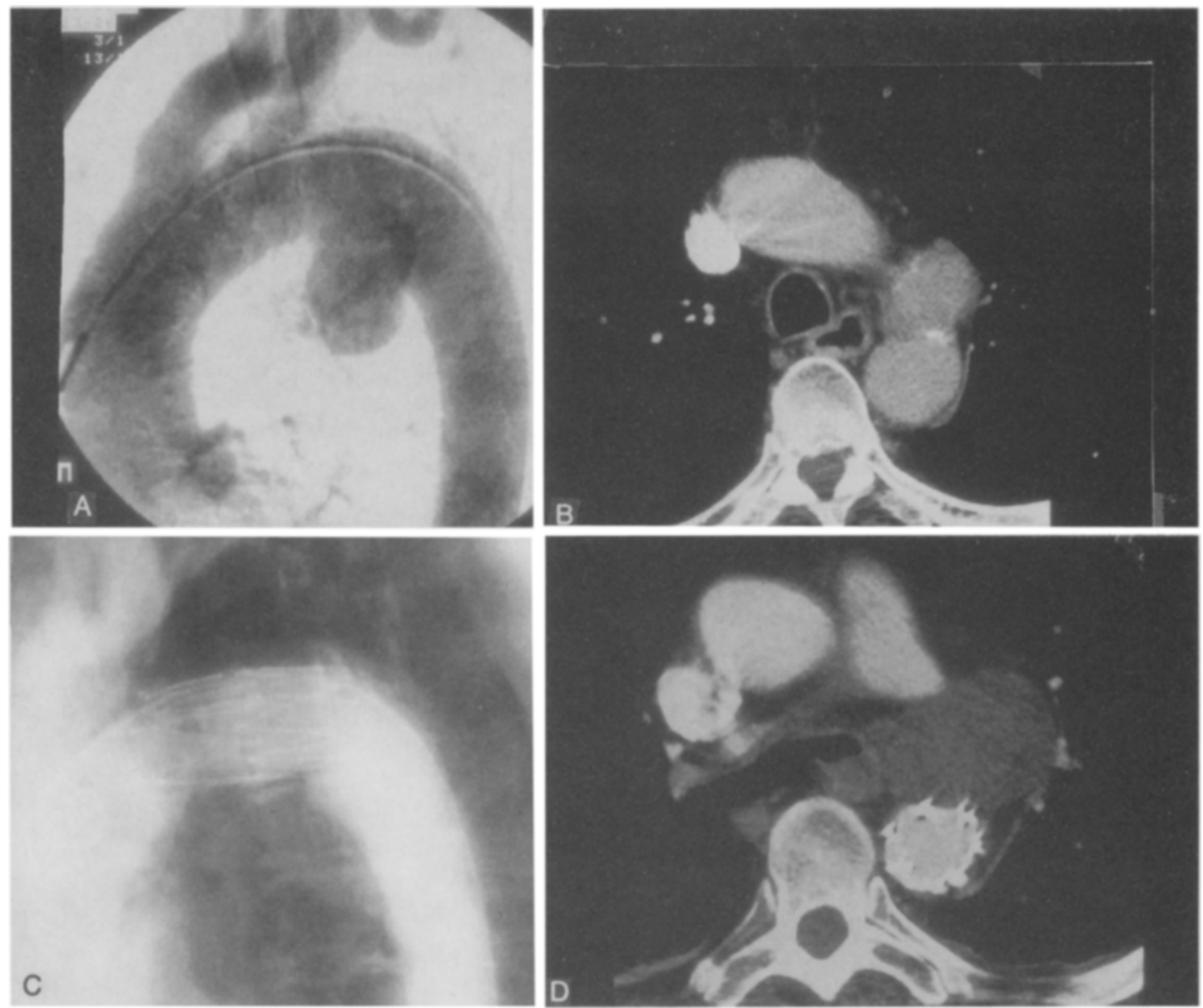

Fig. 2. A, Angiogram demonstrating aneurysm of the ductus diverticulum in a 77-year-old man. B, Computed tomographic scan of same patient, with aneurysm noted on underside of arch. $\mathbf{C}$, After stent-graft deployment, the aneurysm no longer fills with contrast medium. D, Contrast-enhanced computed tomographic scan after stent-graft deployment confirms absence of aneurysm filling.

with stent-graft insertion through a $10 \mathrm{~mm}$ side limb. A failed femoral artery cannulation prompted a retroperitoneal approach to the aorta in five patients, to a previous aortic graft in two patients, and to the iliac artery in two patients (Table III). As with any atherosclerotic patient population, other medical problems were frequently encountered (Table IV): hypertension was present in 36, diabetes mellitus in eight, renal insufficiency in six, and congestive heart failure in nine. Thirty-three patients were or had been smokers, and chronic obstructive pulmonary disease was present in 22 . Twelve had undergone previous cardiac operations, and 16 had a history of one or more previous aortic aneurysmal repairs. Twenty-two patients were considered to be reasonable operative candidates. The remaining 22 patients $(50 \%)$ were deemed ineligible for a conventional thoracotomy approach; 16 on the basis of severe chronic obstructive pulmonary disease (forced expiratory volume in 1 second $<700 \mathrm{ml}$ ), with or without accompanying coronary artery disease, congestive heart failure, renal insufficiency, and advanced age or chronic debility; three because of multiple previous thoracotomies, including two with a previous attempt at aneurysmal repair that had been aborted; and three additional patients because of obesity, history of prolonged respiratory insufficiency, severe congestive heart failure, renal insufficiency, severe coronary artery disease, or a combination of these factors (Table III).

\section{Results}

Stent deployment was technically successful in all cases, although multiple stents were required in 16 patients $(36 \%)$. Complete aneurysmal thrombosis was achieved immediately in 36 patients (81\%). There were three deaths in the perioperative period. The first death occurred on postoperative day 27 , after successful graft deployment, as a result of 
Table II. Patient characteristics of nonoperative candidates

\begin{tabular}{ccc}
\hline Condition & No. & $\%$ \\
\hline $\begin{array}{l}\text { COPD with or without CAD, CHF, } \\
\text { renal insufficiency }\end{array}$ & 16 & 36 \\
Multiple previous thoracotomies & 3 & 7 \\
$\begin{array}{l}\text { Previous ventilatory dependency, } \\
\text { CHF, CAD, renal insufficiency }\end{array}$ & 3 & 7 \\
ToTAL & 22 & 50 \\
\hline
\end{tabular}

$C O P D$, Chronic obstructive pulmonary disease; $C A D$, coronary artery disease; $C H F$, congestive heart failure.

Table III. Arterial access

\begin{tabular}{lrr} 
& No. & $\% *$ \\
\hline Femoral artery & 23 & 52 \\
Retroperitoneal approach $\dagger$ & 9 & 20 \\
Iliac artery & 2 & 5 \\
Aorta & 5 & 11 \\
Aortic graft & 2 & 5 \\
Concomitant abdominal aortic & 12 & 27 \\
aneurysm repair $\$$ & &
\end{tabular}

${ }^{*}$ Percentages do not add to 100 because of rounding.

†Failed femoral approach necessitated retroperitoneal approach in nine patients.

$\$$ Twelve patients underwent thoracic stent-graft placement concomitantly with abdominal aortic aneurysm repair through a $10 \mathrm{~mm}$ side limb.

progressive renal dysfunction and multisystem organ failure in an elderly (84-year-old) Asian man. The second death occurred on postoperative day 31 after combined rerepair of a suprarenal aneurysm and thoracic stent-graft placement. This patient had a postoperative coagulopathy and ultimately died of renal and respiratory insufficiency and fungal septicemia. The third operative death occurred on the first postoperative day, after disruption of a previously placed aortoiliac graft during sheath advancement. Emergency receliotomy with concomitant thoracic stent-graft placement resulted in a hemorrhagic diathesis, with subsequent inability to maintain adequate ventilation. Of note, none of these three patients were considered candidates for postoperative day conventional aneurysmal repair. There were two early instances of paraplegia. The first occurred in a white woman whose procedure was not performed according to protocol; no cardiopulmonary bypass was on standby. Stent-graft deployment resulted in transient aortic occlusion, and distal aortic hypotension persisted for longer than 60 minutes. Renal and hepatic insufficiency gradually resolved in the perioperative period, and the neurologic deficit gradually improved. This patient
Table IV. Patient characteristics

\begin{tabular}{lrc}
\hline & $N o{ }^{*}$ & $\%^{*}$ \\
\hline History of tobacco use & 33 & 75 \\
Hypertension & 36 & 82 \\
COPD & 22 & 50 \\
Diabetes mellitus & 8 & 18 \\
Renal insufficiency & 6 & 14 \\
\hline
\end{tabular}

COPD, Chronic obstructive pulmonary disease.

*Number of patients does not add to 44 and percentages do not add to 100 because, as with most aneurysm populations, these patients harbored multiple cardiovascular risk factors.

subsequently became unavailable for follow-up. A second instance of paraplegia occurred in the same patient described as the second operative death. After rerepair of a suprarenal aneurysm and deployment of a thoracic stent-graft $12.5 \mathrm{~cm}$ in length, this white man was noted to be paraplegic. Whether distal embolization or intercostal artery interruption was responsible for this spinal cord injury remains unknown.

Difficulties related to imprecise graft deployment in juxtaposition to the left subclavian artery occurred in five patients. In one patient, thrombosis of the proximal subclavian artery developed perioperatively after partial coverage of the subclavian ostium with the stent-graft, but this patient has remained free of symptoms for 7 months. One patient required subclavian-to-carotid transposition for symptomatic subclavian occlusion 3 days after stent-graft placement. Another patient had a separate subclavian artery ostial stent placed to relieve impingement by the aortic stent-graft. Two patients underwent subclavian-to-carotid transposition procedures during the same operation but before stent-graft deployment, to create a more adequate proximal neck.

Subintimal aortic dissections were created in two patients during stent-graft placement. One was covered by a more proximally placed stent-graft. The second patient has been stable for longer than 4 months, and follow-up 6-month computed tomographic scan is pending.

Another patient with a chronic type A dissection extending distally to the aortic bifurcation had a stent-graft placed for management of a contained rupture of the false lumen. Although this was successful, the patient required subsequent descending aortic exclusion 3 months after stent-grafting for continued thoracic pain.

As previously noted, immediate aneurysmal exclusion was noted in 36 patients. Twenty-seven of these patients required only a single stent-graft, seven required two stent-grafts, and two required 
three stent-grafts. Late aneurysmal thrombosis was attained in an additional three patients with coiling in one patient and an additional stent-graft in two patients, for an overall aneurysmal thrombosis rate of $88 \%$ (39/44).

There were two late deaths. The first occurred $3^{1 / 2}$ months after operation from aneurysmal rupture into the patient's esophagus. This patient was known to have had an early failure of aneurysmal thrombosis as the result of a midgraft leak and was scheduled for early return for further treatment. Unfortunately, a small-bowel obstruction unrelated to his stent-graft procedure developed, and the patient was hospitalized at another institution for an extended period. During that hospitalization, aortic expansion was noted on a plain chest roentgenogram but was not further evaluated. Exsanguinating hemorrhage developed after subsequent aneurysmal expansion at its distal extent and erosion into the esophagus. This case emphasizes the importance of complete aneurysmal exclusion. A second patient died suddenly 12 months after stent-graft placement, presumably of an aneurysm-related hemorrhage; complete aneurysmal exclusion had been noted before discharge. Actuarial survival at 35 months was $82 \%$ (Fig. 3).

All other patients $(n=37)$ are alive and well at last follow-up, a mean of 12.6 months after stent grafting. There have been no documented instances of late graft thrombosis, migration, infection, or embolization. In 15 patients followed up for longer than 1 year, one aneurysm continues to be incompletely thrombosed, nine aneurysmal sacs are unchanged in dimension, and five aneurysms have decreased in size. One patient acquired a new proximal leak 35 months after the initial stent-graft procedure. Percutaneous management with radiographic embolization has eliminated that leak. All survivors remain pain free.

\section{Discussion}

Endovascular aneurysmal repair has recently been popularized as a treatment of abdominal aortic aneurysms and other peripheral arterial aneurysms. There appear to be two major prerequisites for such repair, vascular access of sufficient size and limited tortuosity and local anatomic suitability. Specifically, suitability entails a relatively straight segment of the aorta and the absence of nearby important side branches. Although curved segments of the distal aortic arch were able to be successfully grafted in this study, these procedures were associated with
(\%)

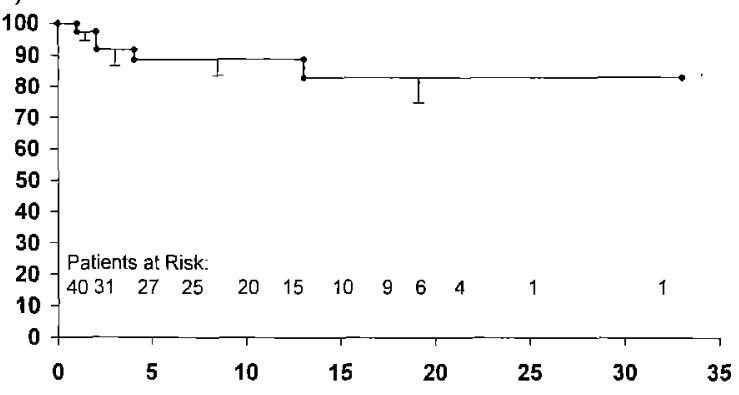

Fig. 3. Actuarial survival after aortic stent-graft placement. Horizontal axis, survival in months; vertical axis, percent survival.

most of the difficulties with maldeployments and aortic dissections. Proximity of the subclavian artery compromising an adequate proximal neck also caused problems, necessitating operative correction in four patients. Later in our experience, catheter access was first gained through the brachial artery if graft deployment was planned in close proximity to the left subclavian artery, with a guide wire traversing the subclavian artery into the aorta. This allowed subsequent stent placement to maintain subclavian patency. There were no instances of grafts compromising the celiac axis or superior mesenteric artery, nor were there any instances of paraplegia attributable solely to occlusion of patent intercostal arteries. If the expected graft location was sufficiently close to an intercostal artery thought to be important for distal spinal cord blood supply, a segment of uncovered stent was attached distally to anchor the prosthesis, thus not compromising patent critical intercostal flow. Because the exact etiology of paraplegia after operative repair is multifactorial, the effect endoluminal grafting will have on paraplegia rates is unknown. Although the opportunity for intercostal reimplantation is not possible, there is less ischemic time and it is hoped that the incidence of paraplegia will be no worse than that associated with the open repair.

Operative mortality for this small series of select patients was $7 \%(n=3)$. Multiple authors have reported mortality rates ranging from $2 \%$ to $15 \%$, although most included emergency as well as elective procedures. Fully $50 \%$ of our patients were not considered to be operative candidates, however, so this mortality rate may not be excessively high.

Stent-graft sizing was determined from a preoperative spiral computed tomographic scan. Stent- 
graft diameter was always oversized by 1 to $3 \mathrm{~mm}$ to achieve a tight friction seal, theoretically allowing continued stent-graft radial expansion should aortic dilatation in either "neck" region progress in the future.

Occlusive aortoiliac disease is a relative contraindication for femoral access, although some have proposed balloon angioplasty to a size sufficient to pass the $26 \mathrm{~F}$ sheath. Planned femoral access failed in five patients, two female and three male. A retroperitoneal approach to the iliac artery or aorta was successful in four of these patients, but one patient had disruption of an aortoiliac graft that required emergency repair, probably contributing to that patient's early operative death. Some anatomic situations were obviously more favorable than others. Placement of a thoracic stent-graft concomitantly with retroperitoneal abdominal aortic aneurysm repair, through a $10 \mathrm{~mm}$ side arm, was generally simple and quick. Lesions in the relatively straighter middescending thoracic aorta were generally more amenable to stent-graft repair than were lesions in the distal arch or lesions associated with a serpentine course of the aorta through the diaphragm. Penetrating ulcers or saccular aneurysms were also generally more easily repaired than large fusiform aneurysms associated with tortuous aortas. We currently believe that chronic aortic dissections are generally not approachable because the cylindrical graft would not be expected to effect a seal in the nonoval true lumen. In the acute type $\mathrm{B}$ aortic dissection, however, stent-graft coverage of the proximal entry point may be possible, with subsequent thrombosis of the false lumen as suggested by previous experiments in a canine model. ${ }^{10}$ We have also enjoyed some limited experience with acute traumatic aortic disruption at the isthmus. Further experience and follow-up will clarify the utility of this application. Finally, we have used this approach for the second stage of an elephant trunk repair for diffuse aneurysmal involvement of the entire thoracic aorta, allowing completion of this procedure in a much shorter time course.

\section{Conclusion}

Further investigation and follow-up are clearly necessary to validate this approach for aneurysmal repair. Long-term evaluation is mandatory to ascertain the efficacy of these stent-grafts in preventing further aneurysmal dilatation and rupture. Graft stability and durability in the long term are also essential prerequisites before widespread use is en- tertained. In its present state, graft deployment is inexact and relatively uncontrolled. Improved methods of accurate graft placement, miniaturization, and capabilities for graft recovery must be developed before this technique can be widely applied. For patients with adequate ileofemoral access, relatively straight aortic segments, and no critical arterial branches, this technology may offer an acceptable method for repair of aneurysms of the descending thoracic aorta. Whether the perceived advantages of reduced operative time, blood loss, hospital stay, overall complications, and mortality will be confirmed awaits further study. Indeed, long-term studies of large numbers of patients over decades are necessary to determine the ultimate utility of transluminally placed endovascular stentgrafts.

\section{REFERENCES}

1. Swan H, Maaske C, Johnson ME, Grover R. Arterial homografts: II. Resection of thoracic aortic aneurysm using a stored human arterial transplant. Arch Surg 1950;61:732-7.

2. DeBakey ME, Cooley DA. Successful resection of aneurysm of the thoracic aorta and replacement by graft. JAMA 1953;152:673-6.

3. Bickerstaff LK, Pairolero PC, Hollier LH, et al. Thoracic aortic aneurysms: a population-based study. Surgery 1982;92: 1103-8.

4. Pressler V, McNamara JJ. Thoracic aortic aneurysm: natural history and treatment. J Thorac Cardiovasc Surg 1980;79: 489-98.

5. Moreno-Cabral CE, Miller DC, Mitchell RS, et al. Degenerative and atherosclerotic aneurysms of the thoracic aorta. J Thorac Cardiovasc Surg 1984;88:1020-32.

6. Borst HG, Jurmann M, Buhner B, Laas J. Risk of replacement of descending aorta with a standardized left heart bypass technique. J Thorac Cardiovase Surg 1994;107:126-33.

7. Verdant A. Descending thoracic aortic aneurysms: surgical treatment with the Gott shunt. Can J Surg 1992;35:493-6.

8. Parodi JC, Palmaz JC, Barone HD. Transfemoral intraluminal graft implantation for abdominal aortic aneurysms. Ann Vase Surg 1991;5:491-9.

9. Dake MD, Miller DC, Semba CP, et al. Transluminal placement of endovascular stent-grafts for the treatment of descending thoracic aortic aneurysms. N Engl J Med 1994;331: 1729-34.

10. Slonim SM, Semba CP, Miller DC, et al. Aortic dissection: percutaneous management of ischemic complications with endovascular stents and balloon fenestration. J Thorac Cardiovase Surg [In press].

\section{Discussion}

Dr. Richard P. Anderson (Seattle, Wash.). This is a new field for most of us, and if any people in this room have had direct experience with stent-grafting of abdominal aortic aneurysms, I hope they will comment from firsthand knowledge, which I lack. These developments, 
for the most part, have appeared in the vascular literature, not in the cardiovascular literature.

Charles Dotterer of the University of Oregon, whom many of you knew, was an extremely innovative interventional radiologist and was probably the first to successfully implant experimentally a metallic stent into an animal's artery and get long-term patency. This was reported in 1969 . In the 20 years thereafter, the idea that endovascular stenting might be useful in variety of applications was widely recognized, and several patents have been issued by the U.S. patent office.

Stents come in various configurations. There is the self-expanding stent, such as Dr. Mitchell has described here, which is similar to the stent used in biliary tract operations. There are also malleable stents that require expansion of a balloon catheter within them for deployment. Those of us who do cardiac operations are familiar with the coronary artery stents that are now so prevalent. Cardiologists tend to use them for sealing dissections or intimal flaps that have been created during angioplasty.

The concept of joining a metallic stent to a vascular prosthesis for aneurysmal repair was first applied clinically in the abdominal aorta, and during the last 4 years or so there have been several reports in the vascular literature of trials involving small numbers of patients. This technique has been employed with about the same degree of success and with the same kind of problems that we have heard described here. This is the first clinical report of which I am aware, however, of the use of an endovascular stent to repair thoracic aneurysms. It is also probably the largest single reported series of patients with endovascular stent-grafting. The results sound promising-7\% mortality associated with thoracic aortic aneurysm-particularly when you consider the risk factors in the group Dr. Mitchell has shown us, half of whom were very high-risk patients. Ninety percent aneurysmal thrombosis shortly after stent deployment is good because you must have aneurysmal thrombosis for long-term success. If you do not, and the interior of the aneurysm is still subjected to arterial pressure, the aneurysm may expand and ultimately rupture, as it did late in one of these patients.

With that as a background, I have a couple of questions. First, because leakage around the stent is a problem that may lead to either failure of thrombosis or late reentry of the circulation into the aneurysmal sac, how difficult is it to achieve a complete seal around this stent? If you cannot achieve this at the time of deployment, what other options do you have? Or do you accept inability to seal a leak at initial deployment?

Dr. Mitchell. This has been an evolutionary series, and we have learned a lot. We think that it is imperative to get a complete seal so that there is no filling of the aneurysmal sac. At least one of our late deaths was certainly instructive, insofar as the necessity of eliminating aneurysmal opacification is concerned. This patient's leak was just distal to the distal stent. I think one of the limitations of this technology is that it is impossible to assess the exact extent of the aneurysm as carefully as during operation. As you saw, however, 29 patients had exclusion of their aneurysm with a single stent, fully another 13 required only two stents, and only two patients required three stents. There have been some leaks even after that. One patient has required interventional coiling of a proximal leak. In fact, just before our departure for this meeting, our very first patient, who has been followed up now for almost 3 years; acquired a new small leak proximally. $\mathrm{He}$ is going to undergo coil placement when we get back.

Dr. Anderson. With respect to that case, I recall that the early patients in the series were those not at high risk. They were patients who would have otherwise been operative candidates. Is that correct?

Dr. Mitchell. That is correct. The first four or five patients were all operative candidates.

Dr. Anderson. At this point, how are you going to manage this patient's condition? Are you going to declare defeat and go in and operate and resect his aneurysm, or are you going to attempt another endovascular procedure?

Dr. Mitchell. This patient is pretty insistent, despite being pretty healthy, about avoiding an operation. We are therefore going to attempt to put coils in when we get back to Stanford.

Dr. Anderson. Tell him that an operation will be a lot more pleasant if his aneurysm is not ruptured when it is performed.

I note that you mentioned that you were very careful to protect these patients during the initial part of the study by various means-procedures were done in the operating room with general anesthesia, pump standby, operating team, and so on. Yet you have now told us that there was no acute need to convert any of these procedures to open operation. Have you backed off on your precautions, and would you perform procedures in the radiology suite now?

Dr. Mitchell. No. We are not going back off on those criteria. The one patient who had paraplegia could have had some significant amelioration of these complications had distal bypass been instituted instantly, and so I think we will continue to have cardiopulmonary bypass available and have surgeons standing by in the operating room.

Dr. Anderson. Would you comment then on the recent trial of stent-grafting of abdominal aortic aneurysms? I understand that the investigators aborted the trial about halfway through. Could you comment on that in light of your own experience and discuss whether the same problems might not arise in your series?

Dr. Mitchell. The stent-graft repair to which you are referring is with the stent manufactured by Endovascular Technologies, Inc. (Menlo Park, Calif.), which is for infrarenal aneurysmal repair. That was a stent commercially manufactured specifically for this graft repair and is different in many aspects from the device used in our study. The first difference is that they used hooks to attach their device to the aortic wall, and one of the fracture points happened to be in that radius of curvature. Subsequently, they have developed a model to test it, and they can now duplicate that failure. They think there is a fatigue problem at that curvature. That has 
been modified with an increased radius of curvature, and they have not seen subsequent fractures. The other site of fracture was at a weld site of this hook to the actual stent, and that has been eliminated in the subsequent design iterations of this device. The modified device is currently back in consideration at the
Food and Drug Administration, and clinical trials are scheduled to resume in early November of 1995.

Dr. Anderson. I commend you not only on the results that you have achieved but on your courage in attempting this very innovative technique. I am certain that it will have application in selected patients in the future. 